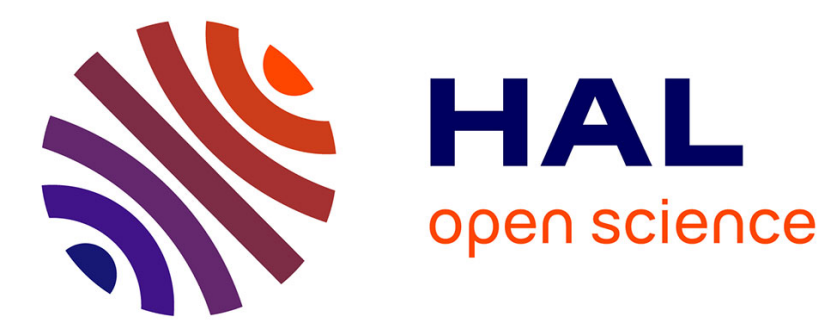

\title{
Observer-based approach for Synchronization of a Time-Delayed Chua's Circuit
}

\author{
Estelle Cherrier, Mohamed Boutayeb, José Ragot
}

\section{To cite this version:}

Estelle Cherrier, Mohamed Boutayeb, José Ragot. Observer-based approach for Synchronization of a Time-Delayed Chua's Circuit. May 2005, 4 p. hal-00118567

\section{HAL Id: hal-00118567 \\ https://hal.science/hal-00118567}

Submitted on 5 Dec 2006

HAL is a multi-disciplinary open access archive for the deposit and dissemination of scientific research documents, whether they are published or not. The documents may come from teaching and research institutions in France or abroad, or from public or private research centers.
L'archive ouverte pluridisciplinaire HAL, est destinée au dépôt et à la diffusion de documents scientifiques de niveau recherche, publiés ou non, émanant des établissements d'enseignement et de recherche français ou étrangers, des laboratoires publics ou privés. 


\section{Observer-based approach for Synchronization of a Time-Delayed Chua's Circuit}

\author{
Estelle Cherrier \\ CRAN UMR CNRS 7039 \\ INPL, 2 Avenue de la Forêt de Haye \\ 54516 Vandoeuvre-lès-Nancy Cedex, France \\ cherrier@eavr.u-strasbg.fr
}

\author{
Mohamed Boutayeb \\ LSIIT UMR CNRS 7005 \\ ULP, Pôle API, Bd Sébastien Brandt \\ BP 10413 \\ 67412 Illkirch, France \\ Mohamed.Boutayeb@ipst-ulp.u-strasbg.fr
}

\author{
José Ragot \\ CRAN UMR CNRS 7039 \\ INPL, 2 Avenue de la Forêt de Haye \\ 54516 Vandoeuvre-lès-Nancy Cedex, France \\ Jose.Ragot@ensem.inpl-nancy.fr
}

\begin{abstract}
In this note, we develop a reduced-order observer-based approach for synchronization of time-delayed chaotic systems. Indeed, in a recent work, Wang et al. proposed an interesting technique to enhance the complexity of the chaotic behavior of the standard Chua's circuit, through a time-delay feedback. We provide here a specific solution to ensure the synchronization of this class of nonlinear systems, even in the case of an unknown (or time-varying) delay. Our synchronization scheme relies on the design of a reduced-order observer, whose efficiency is tested on the numerical example provided in [18]. This work is the first step in the design of a secure communications scheme.
\end{abstract}

\section{INTRODUCTION AND PROBLEM FORMULATION}

\section{A. Synchronization of chaotic systems}

Over the last decade, the synchronization of chaotic systems has become a wide field of research activities. The pioneering works [17], [5] have been the starting point of several synchronization schemes. For a long time, chaotic phenomena were considered as perturbations to be avoided. Since chaotic systems do not form a class of nonlinear systems which can be defined explicitly by a model, they are rather characterized by some noticeable properties: chaotic systems exhibit a great sensibility to their initial conditions; they are long-term unpredictable and look like noise, though they are deterministic; their power spectrum is wide and continuous-like ... Before 1990 the extreme sensibility of chaotic systems to initial conditions remained a major drawback, preventing chaotic systems from being exploited in domains such as estimation, control ... The works of Pecora and Carroll have shown that, in spite of this extreme sensibility to initial conditions, two chaotic systems with different initial conditions could synchronize under some conditions detailed in [17], [5].

The main interest of synchronization lies in its application to secure communications [11], [12]. A communication scheme consists of a transmitter and a receiver, linked with some channel. The transmitter is a chaotic generator, it sends a chaotic signal to the receiver, which synchronizes with the transmitter. Once synchronization is achieved, a message can be hidden in the chaotic signal (since chaotic signals look like noise, one can hope that an intruder will not detect the presence of the message "inside" this chaotic signal). Then, under certain conditions, depending on the type of synchronization scheme, the message can be recovered at the receiver end (see [1], [2] for further details on the way to add the message and to recover it). So, synchronization is a key step in a communication scheme.

In this paper, we will only focus on the phenomenon of synchronization of chaotic systems. This is the first step in the design of a secure communication process, which will be developed in a future paper.

There are two main approaches to study synchronization. The first, called the drive-response principle, was found by Pecora and Carroll in 1990 [17]. In this scheme, the transmitter is called the drive system, and the receiver is called the response system. The driving signal is usually some of the transmitter's state variables, and the response system is chosen as a part of the drive system. It has been shown that, if the conditional Lyapunov exponents [19] of the response system are all negative, synchronization occurs. The main limitation of this concept is that the drive signal and the response system are obtained from the drive system, but there is no systematic procedure available to find a good decomposition of the drive system to ensure negative conditional Lyapunov exponents.

This approach is a kind of self-synchronization, and can be opposed to the second approach, called the observer-based synchronization [14], [15]. Indeed, one can see the synchronization as a state estimation problem: given the chaotic transmitter, the receiver can be designed as an observer of this system. Then the receiver and the drive signal must check a property of detectability to ensure synchronization. Since this is a well-studied problem, some procedures are available to design the observer. Consequently, many papers use this observer-based concept to design synchronization schemes for chaotic systems, among which [10], [9], [13], [4].

In this article, we have chosen the second method, based on the theory of nonlinear state estimation, to establish a synchronization scheme for a modified Chua's circuit.

\section{B. A modified Chua's circuit}

In this section, we will study why we have chosen a modified Chua's circuit in our synchronization scheme.

The standard Chua's circuit is an electric circuit (see Fig. 1), well known to exhibit a wide variety of chaotic behaviors [7]. It has been

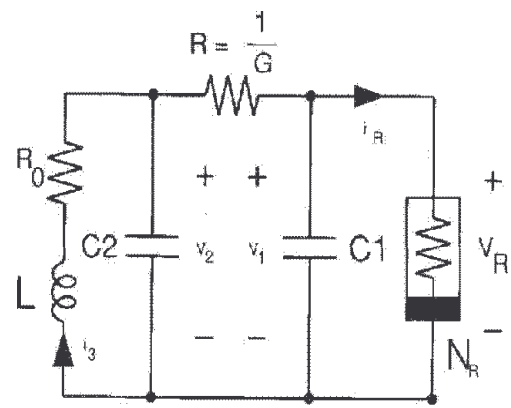

Fig. 1. Chua's circuit

intensively studied, in a theoretical side, as well as in an experimental one, and it has been rigorously proved that it is a chaotic system [7]. Chua's circuit consists of two resistors $R_{0}$ and $R=1 / G$, two 
capacitors $C_{1}$ and $C_{2}$, an inductor $L$, which define the linear part of the dynamic model, and of a nonlinear resistor $N_{R}$ (called Chua's diod), whose piecewise linear characteristic defines the nonlinear part of the dynamic model, given by:

$$
\dot{x}(t)=A x(t)+F(x(t))
$$

with

$$
\begin{gathered}
A=\left(\begin{array}{rrr}
-\frac{G}{C_{1}} & \frac{G}{C_{1}} & 0 \\
\frac{G}{C_{2}} & -\frac{G}{C_{2}} & \frac{1}{C_{2}} \\
0 & -\frac{1}{L} & -\frac{R_{0}}{L}
\end{array}\right) \\
x=\left(\begin{array}{lll}
x_{1} & x_{2} & x_{3}
\end{array}\right)^{T}
\end{gathered}
$$

and

$$
\begin{gathered}
F(x(t))=\left(\begin{array}{ccc}
-\frac{1}{C_{1}} f\left(x_{1}(t)\right) & 0 & 0
\end{array}\right)^{T} \\
f\left(x_{1}\right)=G_{b} x_{1}+\frac{1}{2}\left(G_{a}-G_{b}\right)\left\{\left|x_{1}+E\right|-\left|x_{1}-E\right|\right\}
\end{gathered}
$$

Depending on the values of its parameters, Chua's circuit gives rise to a wide range of chaotic attractors. Therefore synchronization or communication schemes are often tested on this system [16], [13], [4].

Beside, according to [20], double-scroll attractors provide a high level of security, in the sense that they are more robust against the attacks detailed in that article. Complex chaotic signals are also recommended to prevent the unauthorized recovery of the message [10]. Recently, a modified Chua's circuit has been designed in [18]. The proposed method belongs to the "anticontrol" of chaos: by adding a delayed state feedback, a non chaotic system can become chaotic, or the chaotic behavior of a system can be enhanced. All these reasons have prompted us to use this modified Chua's circuit in our synchronization scheme. The dynamic model of this system is [18]:

$$
\dot{x}(t)=A x(t)+F(x(t))+H(x(t-\tau))
$$

with

$$
\begin{gathered}
H(x(t-\tau))=\left(\begin{array}{ccc}
0 & 0 & -\frac{1}{L} h\left(x_{1}(t-\tau)\right)
\end{array}\right)^{T} \\
h\left(x_{1}(t-\tau)\right)=\varepsilon \sin \left(\sigma x_{1}(t-\tau)\right)
\end{gathered}
$$

\section{Problem formulation}

The aim of this work is to design an observer-based synchronization scheme for this class of systems (6). In [6] we have proposed an analytical approach in the case when the delay is known. Büt if the delay is unknown, or time-varying, this last method cannot be applied. Therefore we propose here a reduced-order observer for the system (1), which ensures the synchronization with the modified Chua's circuit, even in the case when the delay is unknown (or varying). Simulation results on the example provided in [18] show the effectiveness of the proposed approach.

This paper is organized as follows. Section II is concerned by the design of the reduced-order observer of Chua's modified circuit (1). Section III ends this work with some numerical simulations.

Notations: Throughout the paper, $I_{n}$ corresponds to the identity matrix of $\mathbb{R}^{n}$, the symbol ()$^{T^{\prime}}$ denotes the vector transpose.

Notice that the function $F$ involved in the nonlinear part of the system satisfies the Lipschitz property, with constant $k=\max \left(G_{a}, G_{b}\right) / C_{1}$ :

$$
\|F(x)-F(y)\| \leq k\|x-y\|, \forall x \text { and } y
$$

\section{DESIGN OF THE REDUCED-ORDER OBSERVER}

In this part, we deal with the design of a reduced-order observer synchronizing with the modified Chua's circuit (6), where the timedelay term $\tau$ is assumed to be unknown, or varying.

To this purpose, we recall the dynamic model to synchronize:

$$
\left\{\begin{array}{l}
\dot{x}(t)=A x(t)+F(x(t))+H(x(t-\tau)) \\
y(t)=C x(t)
\end{array}\right.
$$

Since the delay is assumed to be unknown, the aim is to obtain a new system which does not require this delay. One solution is to eliminate the delay, this can be done by projection. Let $E$ be a matrix orthogonal to the matricial function $H: E H=0$. Such a matrix $E$ exists if and only if ker $H \neq\{0\}$. Besides, assume that the matrix $\left(\begin{array}{c}E \\ C\end{array}\right)$ is of full column rank. From (6), we obtain the following
singular system:

$$
E \dot{x}=E A x+E F(x(t))+E H(x(t-\tau))
$$

With the following definitions:

$$
\begin{aligned}
& \tilde{A}=E A \\
& \tilde{F}=E F
\end{aligned}
$$

The system (10) can be rewritten as:

$$
\left\{\begin{array}{l}
E \dot{x}(t)=\tilde{A} x(t)+\tilde{F}(x(t)) \\
y(t)=C x(t)
\end{array}\right.
$$

Since the matrix $\left(\begin{array}{c}E \\ C\end{array}\right)$ is of full column rank, there exist two matrices $P$ and $Q$ of appropriate dimensions, such that:

$$
\left(\begin{array}{ll}
P & Q
\end{array}\right)\left(\begin{array}{c}
E \\
C
\end{array}\right)=I_{3}
$$

We have then:

$$
\left(\begin{array}{ll}
P & Q
\end{array}\right)=\left(\left(\begin{array}{c}
E \\
C
\end{array}\right)^{T}\left(\begin{array}{c}
E \\
C
\end{array}\right)\right)^{-1}\left(\begin{array}{c}
E \\
C
\end{array}\right)^{T}
$$

We set

$$
z=T x=\left(\begin{array}{l}
z_{1} \\
z_{2}
\end{array}\right)
$$

The reduced-order observer that we propose is of the form:

$$
\left\{\begin{array}{l}
\dot{z}(t)=N z(t)+K y(t)+r(z(t), y(t)) \\
\hat{z}(t)=z(t)+T Q y(t)
\end{array}\right.
$$

where $N, K$ and the function $r$ will be determined hereafter.

Before we give the proof of the convergence of the reduced-order observer, we underline that the third component of the state of the initial system (10) is not taken into account in the design of the observer, so the delay $\tau$ is allowed to be unknown (or varying).

The following theorem provides a sufficient condition for ensuring the synchronization of the reduced-order observer:

Theorem 2.1:

If these conditions are fulfilled:

- detectability condition:

$$
\operatorname{rank}\left(\begin{array}{c}
s E-\tilde{A} \\
C
\end{array}\right)=\operatorname{dim} x, \forall s \geq 0
$$

- there exists a matrix $N$ such that:

$$
N T P E-T P \tilde{A}+K C=0
$$

- the matrix

$$
\left(\begin{array}{c}
T P E \\
C
\end{array}\right)
$$


is non singular

- there exist two symmetric, positive-definite matrices $W$ and $U$ such that

$$
\begin{aligned}
& N^{T} U+U N=-W \\
& W-k \gamma I_{2}>0
\end{aligned}
$$

with $\gamma=2\|U T P\|$

then the function $r(z, y)$ can be defined by :

$$
r(z, y)=T P \tilde{F}(\hat{x})
$$

with

$$
\hat{x}=\left(\begin{array}{c}
T P E \\
C
\end{array}\right)^{-1}\left(\begin{array}{l}
z \\
y
\end{array}\right)
$$

In this case, the reduced-order observer (17) is asymptotically convergent, and $\hat{x} \rightarrow x$.

Proof:

The reduced state estimation error vector is defined by $e=\hat{z}-z$. If we use (14) and (17), we obtain:

$$
\begin{aligned}
e & =z+T Q y-z \\
& =T\left(I_{3}-P E\right) x \\
& =z-T P E x
\end{aligned}
$$

Then, the error dynamics is given by:

$$
\dot{e}=N z+K y+r(z, y)-T P(\tilde{A} x+\tilde{F}(x))
$$

By making use of (13), (17) and (24), we obtain:

$$
\dot{e}=N e+(N T P E-T P \tilde{A}+K C) x+r(z, y)-T P \tilde{F}(x)
$$

If we make use of (19) and (22), (26) becomes:

$$
\dot{e}=N e+T P(\tilde{F}(\hat{x})-\tilde{F}(x))
$$

In order to obtain $N$ stable, we must find a symmetric positive definite matrix $U$ such that:

$$
N^{\prime} U+U N=-W
$$

with $W>0$.

Consider then the Lyapunov function $V=e^{T} U e$. The derivative of $V$ along the trajectories of (27) is given by:

$$
\begin{aligned}
\dot{V}= & e^{T}\left(N^{T} U+U N\right) e+e^{T} U T P(\tilde{F}(\hat{x})-\tilde{F}(x)) \\
& +(\tilde{F}(\hat{x})-\tilde{F}(x))^{T}(T P)^{T} U e
\end{aligned}
$$

(29) can be upper bounded by:

$$
\dot{V} \leq-e^{T} W e+2\|U T P\|\|e\|^{2}
$$

where $k$ is the Lipschitz constant of $\tilde{F}$.

Since $\gamma=2\|U T P\|$, we obtain finally:

$$
\dot{V} \leq-e^{T}\left(W-k \gamma I_{2}\right) e
$$

Now, we give a procedure to build the reduced-order observer of the modified Chua's circuit (10).

First we choose the observation matrix:

$$
C=\left(\begin{array}{lll}
0 & 1 & 1
\end{array}\right)
$$

Then the matrix $E$, orthogonal to $H$, is computed:

$$
E=\left(\begin{array}{lll}
1 & 0 & 0 \\
0 & 1 & 0
\end{array}\right)
$$

It is easy to check that the detectability condition (18) is fulfilled.

The next step consists in finding a convenient matrix $N$. Notice that by using (14) and (19), we obtain:

$$
N T-T P \tilde{A}=M C
$$

with

$$
M=N T Q-K
$$

Besides, by the use of (20), there exist $L_{1}$ and $L_{2}$ of appropriate dimensions such that:

$$
\left(\begin{array}{c}
T P E \\
C
\end{array}\right)^{-1}=\left(\begin{array}{ll}
L_{1} & L_{2}
\end{array}\right)
$$

If we multiply (34) by $L_{2}$, (36) leads to:

$$
M=N T L_{2}-T P \tilde{A} L_{2}
$$

Now, if we multiply (19) by $L_{1}$, with (14), we obtain:

$$
N=T P \tilde{A} L_{1}
$$

We introduce a matrix $R$ such that $\left(\begin{array}{c}R \\ C\end{array}\right)$ is invertible, and $\left(\begin{array}{c}T P E \\ C\end{array}\right)$ and $\left(\begin{array}{c}R \\ C\end{array}\right)$ are linked by (see [3]):

$$
\left(\begin{array}{c}
T P E \\
C
\end{array}\right)=\left(\begin{array}{cc}
I_{2} & -F \\
0 & 1
\end{array}\right)\left(\begin{array}{l}
R \\
C
\end{array}\right)
$$

Then (14) and (39) give:

$$
T=R+S C
$$

with

$$
S=T Q-F
$$

If we replace (40) in (38), we obtain:

$$
N=R P \tilde{A} L_{1}-S C P \tilde{A} L_{1}
$$

Notice that if the pair $\left(R P \tilde{A} L_{1}, C P \tilde{A} L_{1}\right)$ is detectable, then by a pole placement, the matrix $S$ can be chosen so that $N$ is stable. It has been proved in [8] that this condition of detectability is equivalent to the condition (18).

We apply this procedure to the example of the modified Chua's circuit (6).

We can choose

$$
R=\left(\begin{array}{lll}
1 & 0 & 0 \\
0 & 1 & 0
\end{array}\right)
$$

We use (36), (39), and we get:

$$
L_{1}=\left(\begin{array}{l}
R \\
C
\end{array}\right)^{-1}\left(\begin{array}{c}
I_{2} \\
0
\end{array}\right)=\left(\begin{array}{rr}
1 & 0 \\
0 & 1 \\
0 & -1
\end{array}\right)
$$

We apply (42), where $S$ is chosen so that $N$ is stable: since $C P \tilde{A} L_{1}=0$, (42) is reduced to

$$
N=R P \tilde{A} L_{1}=\left(\begin{array}{rr}
-\frac{G}{C_{1}} & \frac{G}{C_{1}} \\
\frac{G}{C_{2}} & -\frac{G+1}{C_{2}}
\end{array}\right)
$$

By studying the determinant of $N$, it is easy to show that it is a stable matrix. 
So, $S$ can be chosen arbitrarily, for example $S=\left(\begin{array}{ll}1 & 0\end{array}\right)^{T}$. We set

$$
\begin{aligned}
T & =R+S C=\left(\begin{array}{lll}
1 & 1 & 1 \\
0 & 1 & 0
\end{array}\right) \\
F & =T Q-S=\left(\begin{array}{l}
0 \\
0
\end{array}\right) \\
L_{2} & =\left(\begin{array}{c}
R \\
C
\end{array}\right)^{-1}\left(\begin{array}{c}
F \\
1
\end{array}\right)=\left(\begin{array}{c}
0 \\
0 \\
1
\end{array}\right) \\
M & =N T L_{2}-T P \tilde{A} L_{2}=\left(\begin{array}{c}
-\frac{G}{C_{1}} \\
\frac{G-1}{C_{2}}
\end{array}\right) \\
K & =N T Q-M=\left(\begin{array}{c}
0 \\
\frac{1}{C_{2}}
\end{array}\right)
\end{aligned}
$$

The Lyapunov equation (28) can easily be solved numerically, and this ends the design of the proposed synchronization scheme

\section{Numerical Simulations}

The numerical values of the parameters of Chua's modified circuit are those defined in as in [18], they ensure a chaotic behavior with a double-scroll attractor:

$$
\begin{aligned}
& G=1 / 1950, C_{1}=10^{-8}, C_{2}=10^{-7}, L=18.68 .10^{-3}, R_{0}=16, E=1, \\
& G_{a}=-0.75 .10^{-3}, G_{b}=-0.41 .10^{-3}, \tau=0.001, \varepsilon=0.2, \sigma=0.5
\end{aligned}
$$

In this example, the delay $\tau$ is fixed to ensure a chaotic behavior. We choose the following initial conditions for the real system and for the observer:

$$
\begin{aligned}
& x_{0}=\left(\begin{array}{lll}
1 & 1 & 0
\end{array}\right)^{T} \\
& z_{0}=\left(\begin{array}{ll}
2 & 0
\end{array}\right)^{T}
\end{aligned}
$$

The numerical integrations are realized with an method Euler, with step size $10^{-7}$ seconds. The figure 2 shows the trajectories of the components of the real state, and the corresponding components of the reconstructed state, and the interval of simulation is $[0,0.01]$ seconds. Our synchronization scheme is very efficient, the synchronization error is equal to zero very fast. The figure 3 shows a zoom of the top curve of Figure 2 during the interval [0,0.001] seconds. A lack of place has prevented us from analyzing the robustness of our synchronization scheme towards channel noise. Our future work will consist in developing a secure communications scheme, based on the designed reduced-observer.

\section{CONCLUSION}

In this work, we addressed the problem of synchronization of a modified Chua's circuit, which belongs to a class of non-linear
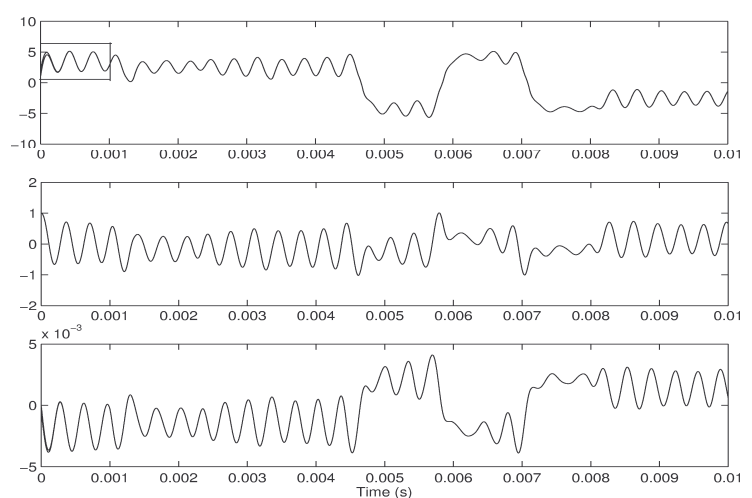

Fig. 2. The three components of the real state and the reconstructed state

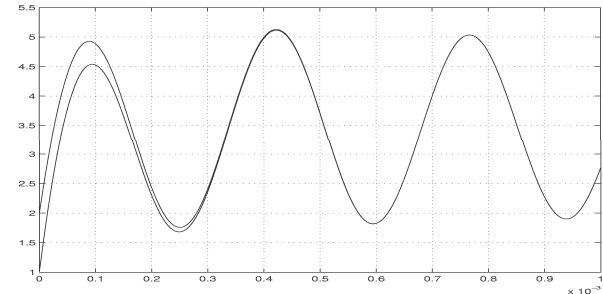

Fig. 3. Zoom on the reconstruction of the first component of the state

systems with a time delay. We have designed an observer-based scheme for synchronization, even when the delay is unknown. This ensures a fast synchronization with the delayed Chua's circuit, which has been tested on numerical simulations.

\section{REFERENCES}

[1] "Special Issue on Chaos Synchronization and control : Theory and Applications," IEEE Trans. Circuit Syst. I, vol. 44, no. 10, pp. 8531039, 1997.

[2] "Special Issue on Control of Chaos and Synchronization," Sys. \& Control Letters, vol. 31, pp. 259-322, 1997.

[3] M. Boutayeb and M. Darouach, "Observers design for non linear descriptor systems," in Proceedings of the 34rd IEEE Conference on Decision and Control,, 1995.

[4] M. Boutayeb, M. Darouach, and H. Rafaralahy, "Generalized StateSpace Observers for Chaotic Synchronization and Secure Communication," IEEE Trans. Circuit Syst. I, vol. 49, no. 3, pp. 345-349, 2002.

[5] T. Carroll and L. Pecora, "Synchronizing Chaotic Circuits," IEEE Trans. Circuit Syst., vol. 38, no. 4, pp. 453-456, 1991.

[6] E. Cherrier and M. Boutayeb, "Observer-based approach for synchronization of modified chua's circuit," in Proceedings of NOLCOS, IFAC Symposium on Nonlinear Control Systems, Stuttgart, Germany, 2004.

[7] L. Chua, C. Wu, A. Huang, and G.-Q. Zhong, "A Universal Circuit for Studying and Generating Chaos-Part I and Part II," IEEE Trans. Circuit Syst. I, vol. 40, no. 10, pp. 732-761, 1993.

[8] M. Darouach and M. Boutayeb, "Design of Observers for Descriptor Systems," IEEE Trans. Automatic Control, vol. 40, no. 7, pp. 13231327, 1995.

[9] M. Feki, "Observer-based exact synchronization of ideal and mismatched chaotic systems," Phys. Lett. A, vol. 309, pp. 53-60, 2003.

[10] G. Grassi and S. Mascolo, "Nonlinear Observer Design to Synchronize Systems via a Scalar Signal," IEEE Trans. Circuit Syst. I, vol. 40, no. 10. pp. 640-656, 1997.

[11] G. Kolumbán, M. P. Kennedy, and L. O. Chua, "The Role of Synchronization in Digital Communications Using Chaos - Part I : Fundamentals of Digital Communications," IEEE Trans. Circuit Syst. I, vol. 44, pp. 927-936, 1997.

[12] _ . "The Role of Synchronization in Digital Communications Using Chaos - Part II : Chaotic Modulation and Chaotic Synchronization," IEEE Trans. Circuit Syst. I, vol. 45, pp. 1129-1140, 1998.

[13] T.-L. Liao and N.-S. Huang, "An Observer-Based Approach for Chaotic Synchronization with Applications to Secure Communications," IEEE Trans. Circuit Syst. I, vol. 46, no. 9, pp. 1144-1150, 1999.

[14] O. Morgül and E. Solak, "Observer based synchronization of chaotic systems," Physical Review E, vol. 54, no. 5, pp. 4803-4811, 1996.

[15] H. Nijmeijer and I. Mareels, "An Observer Looks at Synchronization," IEEE Trans. Circuit Syst. I, vol. 44, no. 10, pp. 882-890, 1997.

[16] M. Ogorzalek, "Taming Chaos - Part I : Synchronization," IEEE Trans. Circuit Syst. I, vol. 40, no. 10, pp. 693-699, 1993.

[17] L. Pecora and T. Carroll, "Synchronization in chaotic systems," Phys. Rev. Lett., vol. 64, no. 8, pp. 821-824, 1990.

[18] X. Wang, G.-Q. Zhong, K.-S. Tang, K. Man, and Z.-F. Liu, "Generating Chaos in Chua's Circuit via Time-Delay Feedback," IEEE Trans. Circuit Syst. I, vol. 48, no. 9, pp. 1151-1156, 2001.

[19] S. Wiggins, Introduction to Applied Nonlinear Dynamical Systems and Chaos. Springer-Verlag, 1990.

[20] T. Yang, L.-B. Yang, and C.-M. Yang, "Cryptanalysing Chaotic Secure Communications Using Return Maps," Phys. Lett. A, vol. 245, pp. 495510,1998 , 